\title{
The optimal emission reduction rate and price with carbon quota and manufacturer competition
}

\author{
Linghong Zhang ${ }^{1, \mathrm{a}}$, Hao Zhou ${ }^{2, \mathrm{~b}}$ and Wenxing Sun ${ }^{2, \mathrm{c}}$ \\ ${ }^{1}$ Management Science and Engineering Postdoctoral Mobile Station, Shandong Normal University, \\ China \\ ${ }^{2}$ School of Management Science and Engineering, Shandong Normal University, China \\ azhanglinghong2005@126.com, bhouhao226219@126.com, ${ }^{\mathrm{c}} 373253360 @ q q . c o m$
}

Keywords: carbon quota; consumer environmental awareness; manufacturer competition; carbon emission rate

Abstract. With environment quality decreases, consumer's environmental awareness (CEA) increases and more and more manufacturers are willing to produce the product with low carbon emission. At the same time, government employs the carbon quota to stimulate the manufacturer to produce green product. This paper studies how CEA and carbon quota affect the carbon emission rates and prices of the products when there are two competitive manufacturers. We give the explicit expressions of the optimal carbon emission rates and prices and analyze the impact of the size of the manufacturer, the sensitivity of switchovers toward price and CEA. We find that (1) the optimal emission reduction rates increase with CEA and prices but decrease with the emission reduction cost coefficient; (2) manufacturers' profits increase with the increase of the sensitivity of switchovers toward price, CEA and the total carbon quota; (3) manufacturers' retail prices, emission reduction rates and profits are equal when the manufacturers' size are the same; When the sizes of two manufacturers are different, the larger the size of the manufacturer, the greater the profit.

\section{Introduction}

According to previous research, at least $90 \%$ of the effects of global warming are likely to be caused by human activities[1]. Nowadays, with the aggravation of the greenhouse effect, environmental protection has become a social responsibility. Consumers pay more and more attention to environmental protection. The rise of environmental awareness changes consumer behavior[2]. The BBMG Conscious Consumer Report shows that $51 \%$ of Americans are willing to pay more for products with high environmental quality and 67\% agree that it is important to buy products with environmental benefits [3]. Thus manufacturers may obtain higher profits by designing the product with low carbon emissions to attract more consumers.

In order to stimulate more manufacturer to produce product with low carbon emission, the government employs many policies, such as subsidy for the green product, allocation finite carbon quota for each manufacturer, tax to the product with high pollution, and so on. Allocating finite carbon quota for each manufacturer is an significant policy to limit manufacturer's emission. And the carbon trading has been prevalent in the international market. Assume that the government allocate the total carbon, in this paper we mainly investigate the following questions:

( 1 ) How consumer environmental awareness impacts emission reduction rate of the product and manufacturers' profits?

( 2 ) How the carbon quota policy affects the manufacturer's strategy?

In this paper we assume that duopoly manufacturers sells two products with different carbon emission rate, the demand functions are determined by price, carbon emission rate and CEA. The manufacturers need to determine the optimal carbon emission rate and price by balancing the $\mathrm{R} \& \mathrm{D}$ cost and carbon trading revenue. Assume two manufacturers take an action simultaneously: they determine the carbon emission rates and prices at the same time. We solve the Nash equilibrium and give some numerical examples and obtain more management insights. 


\section{Literature review}

Our work is closely related to the research on green product with low carbon emission and manufacturer competition in operations management. In the following, we summarize the recent literature of the two areas.

Firstly, we introduce the literature about carbon emission and government policy. Li et al.(2014) analyzes the impact of carbon subsidy on remanufacturing closed-loop supply chain, they also explores the profits and the carbon emission quantities of three types of a supply chain: forward supply chain, remanufacturing closed-loop supply chain, and RCLSC with the carbon subsidy[4]. Yang and Xiao(2017) study how prices, green levels and expected profits are influenced by channel leadership and governmental interventions. Green level sensitivity and fuzzy degree of parameters are considered[5]. Xu and Xie (2016) deeply research into the difference of supply chain system in economic benefits, environmental protection and social benefits[6]. Zhang et al. (2017) investigate the government's optimal subsidy and tax policies in response to consumer environmental awareness (CEA) and the manufacturers' product selection (generic, green or both) plus quality and pricing decisions[7]. However, above literature did not consider the carbon quota allocation and most of the studies did not explore the manufacturers competition.

The second stream focuses on the impact of competition on supply chain enterprises. Liu et al.(2012) focus on the impact of competition and consumers' environmental awareness on key supply chain players by consider both the production competition between partially substitutable products made by different manufacturer[8]. Zhang et al. (2016) focus on the green supply chain performance in a single manufacturer-retailer setup,they found competition improves supply chain efficiency and manufacturer's profit proportion[9]. Chen et al. (2017) take price and emission sensitive demand into account and incorporate competition between the two rival manufacturers in the demand function[10]. Above studies focus on consumer awareness of environmental, competition in the supply chain, and government policy, but these studies did not take the carbon quota allocation into account.

Therefore, in this paper we will mainly study the impact of manufacturers competition, CEA and carbon quota allocation on green product design, price and manufacturers' profits.

\section{Problem assumptions and model description}

In this paper, we assume that there are two manufacturers, and the size of the manufacturer 1 is denoted by $M_{1}$, the size of the manufacturer 2 is denoted by $M_{2}$. The government allocate the total carbon quota, and each manufacturer's carbon quota is determined by the manufacturer's size and competitor's size. If the manufacturer have the surplus of the carbon quota, he could sell it and obtain the extra revenue; but if the carbon emission exceeds the quota, the manufacturer needs to purchase the carbon quota. Therefore, the manufacturer need to determine the environmental quality and price of each product to balance the cost and revenue.

Assumption 1. The total government carbon quota (denote as $s_{0}$ ) is a constant.

Assumption 2. Assume that each product have two attributes, price (denoted as $p$ ) and emission reduction rate (denoted as $e$ ), influencing consumer demand. Product demand increases with emission reduction rate increases and decreases with price.

Similar to Zhang et al. (2015), we consider that the demand functions for products 1 and 2, denoted by $q_{1}$ and $q_{2}$, possess the following structure:

$$
\begin{aligned}
& q_{1}=a-p_{1}+\theta p_{2}+\tau e_{1}-\tau \theta e_{2} . \\
& q_{2}=a-p_{2}+\theta p_{1}-\tau \theta e_{1}+\tau e_{2} .
\end{aligned}
$$

Where a is initial market potential, $\theta$ is the sensitivity of switchovers toward price. Where $\tau$ represents consumer environmental awareness, $p_{i}$ is the retail price of the product, $e_{i}$ is the emission reduction rate.

Similar to Zhu \& He (2017) [11], we model the R\&D cost for the emission reduction rate $e_{i}$ as an 
increasing quadratic function as $c e_{i}^{2}$, independent of the production volume, and $c$ is a strictly positive cost coefficient. Manufacturer $i$ has the carbon quota $\frac{M_{i}}{M_{1}+M_{2}} s_{0}$, and each manufacturer emits the pollution $M_{i}\left(1-e_{i}\right)$ with the emission reduction rate $e_{i}$. If the manufacturer has the surplus of the carbon quota, he could sell it with price $c_{0}$ and obtain the extra revenue; but if the carbon emission exceeds the quota, the manufacturer needs to purchase the carbon quota.

Hence, the profit functions for manufacturer 1 and 2 , denoted by $\pi_{1}$ and $\pi_{2}$, possess the following structures:

$$
\begin{aligned}
& \pi_{1}=p_{1}\left(a-p_{1}+\theta p_{2}+\tau e_{1}-\tau \theta e_{2}\right)-c e_{1}^{2}+\left(\frac{M_{1}}{M_{1}+M_{2}} s_{0}-M_{1}\left(1-e_{1}\right)\right) c_{0} . \\
& \pi_{2}=p_{2}\left(a-p_{2}+\theta p_{1}-\tau \theta e_{1}+\tau e_{2}\right)-c e_{2}^{2}+\left(\frac{M_{2}}{M_{1}+M_{2}} s_{0}-M_{2}\left(1-e_{2}\right)\right) c_{0} .
\end{aligned}
$$

\section{Model formulation and solution}

In this section, we give the two manufacturers' optimal strategies--the optimal emission reduction rates and the optimal retail prices. Therefore, it mainly includes the following two subsection.

\section{The optimal emission reduction rates}

In this subsection, we first give the optimal emission reduction rate, then discuss the impact of CEA, unit price of carbon emission and cost coefficient of emission reduction of the manufacturer on the optimal emission reduction rate; finally discuss the impact of CEA and the sensitivity of switchovers toward price on the optimal profit of the two manufacturers.

Theorem 1. The optimal emission reduction rates of the products and the expected manufacturer's profit are as follows:

$$
e_{1}^{*}=\frac{p_{1} \tau+M_{1} c_{0}}{2 c}, e_{2}^{*}=\frac{p_{2} \tau+M_{2} c_{0}}{2 c} .
$$

Substitute the Formula (5) into the profit function (3) and (4),

$$
\begin{aligned}
& \pi_{1}^{*}=p_{1}\left(a-p_{1}+\theta p_{2}-\tau \theta \frac{p_{2} \tau+M_{2} c_{0}}{2 c}\right)+\frac{\left(p_{1} \tau+M_{1} c_{0}\right)^{2}}{4 c}+s_{0} c_{0} \frac{M_{1}}{M_{1}+M_{2}}-M_{1} c_{0} . \\
& \pi_{2}^{*}=p_{2}\left(a-p_{2}+\theta p_{1}-\tau \theta \frac{p_{1} \tau+M_{1} c_{0}}{2 c}\right)+\frac{\left(p_{2} \tau+M_{2} c_{0}\right)^{2}}{4 c}+s_{0} c_{0} \frac{M_{2}}{M_{1}+M_{2}}-M_{2} c_{0} .
\end{aligned}
$$

As the Theorem 1 shows, The optimal emission reduction rates of the products and the expected manufacturer's profit changes with many factors, the change is reflected in the Propositions 1-3.

\section{Proposition 1.}

(i) The optimal emission reduction rates $e_{1}^{*}$ and $e_{2}^{*}$ increase with $\tau$ and $c_{0}$;

(ii) The optimal emission reduction rates $e_{1}^{*}$ and $e_{2}^{*}$ decrease with $c$.

\section{Proposition 2.}

(i) When $\theta>\frac{p_{1}}{2 p_{2}}$, and

if $\tau \leq \frac{\left(M_{2} \theta-M_{1}\right) c_{0}}{p_{1}-2 p_{2} \theta}$, then the profit of manufacturer 1 decreases with $\tau$;

if $\tau \geq \frac{\left(M_{2} \theta-M_{1}\right) c_{0}}{p_{1}-2 p_{2} \theta}$, then the profit of manufacturer 1 increases with $\tau$.

(ii) When $\theta>\frac{p_{2}}{2 p_{1}}$, and 
if $\tau \leq \frac{\left(M_{1} \theta-M_{2}\right) c_{0}}{p_{2}-2 p_{1} \theta}$, the profit of manufacturer 2 decrease with $\tau$;

if $\tau \geq \frac{\left(M_{1} \theta-M_{2}\right) c_{0}}{p_{2}-2 p_{1} \theta}$, the profit of manufacturer 2 increase with $\tau$.

\section{Proposition 3.}

(i) The manufacturer 1's profit increases with $\theta$ when $0<M_{2}<\frac{p_{2}\left(2 c-\tau^{2}\right)}{\tau c_{0}}$; the manufacturer 1's profit decreases with $\theta$ when $M_{2} \geq \frac{p_{2}\left(2 c-\tau^{2}\right)}{\tau c_{0}}$.

(ii) The manufacturer 2's profit increases with $\theta$ when $0<M_{1}<\frac{p_{1}\left(2 c-\tau^{2}\right)}{\tau c_{0}}$; the manufacturer 2's profit decreases with $\theta$ when $M_{1} \geq \frac{p_{1}\left(2 c-\tau^{2}\right)}{\tau c_{0}}$.

\section{The optimal retail price}

In the subsection 4.1, we have calculated the optimal emission reduction rates of $e_{1}^{*}$ and $e_{2}^{*}$. In this subsection the manufacturer determines the optimal retail price based on its own optimal emission reduction rate.

Theorem 2. The optimal price are as follows:

$$
\begin{aligned}
& p_{1}^{*}=\frac{-4 a c^{2}(\theta+2)+2 a c \tau^{2}(\theta+1)+2 c_{0} c \tau \theta M_{2}+M_{1}\left[2 c_{0} c \tau\left(\theta^{2}-2\right)+c_{0} \tau^{3}\left(1-\theta^{2}\right)\right]}{4 c^{2} \theta^{2}-16 c^{2}-4 c \tau^{2} \theta^{2}+8 c \tau^{2}+\tau^{4} \theta^{2}-\tau^{4}} . \\
& p_{2}^{*}=\frac{-4 a c^{2}(\theta+2)+2 a c \tau^{2}(\theta+1)+2 c_{0} c \tau \theta M_{1}+M_{2}\left[2 c_{0} c \tau\left(\theta^{2}-2\right)+c_{0} \tau^{3}\left(1-\theta^{2}\right)\right]}{4 c^{2} \theta^{2}-16 c^{2}-4 c \tau^{2} \theta^{2}+8 c \tau^{2}+\tau^{4} \theta^{2}-\tau^{4}} .
\end{aligned}
$$

As the Theorem 2 shows, the optimal price changes with many factors, due to the complexity of the formula (8) and (9), the change of the optimal price will be present in the proposition 5 and section 5 numerical examples.

\section{Proposition 4.}

(i) the optimal retail price of manufacturer 1 increases with the size of the manufacturer 2.

(ii) the optimal retail price of manufacturer 2 increases with the size of the manufacturer 1.

Because of the complexity of the formula(8) and (9), we will study the effect of CEA and the sensitivity of switchovers toward price on the optimal retail price in the numerical examples.

\section{Numerical Examples}

To draw more managerial insights from the theoretical results above, we present the numerical analysis in this section. In this section, we mainly study the effect of CEA, the sensitivity of switchovers toward price and the size of the manufacturer on the retail price, optimal emission reduction rate and optimal profit of the two manufacturers. We assume that initial market potential $a=10$, cost coefficient of emission reduction of the manufacturer is $c=50$, unit price of carbon emission $c_{0}=10$, the government's total carbon quota $s_{0}=40$.

\section{Example 1}

In this section,we study the impact of CEA on the emission reduction rates, retailer price and profits of the two manufacturers. In table 2, we assume that the sensitivity of switchovers toward price $\theta=0.5$, the size of the manufacturer 1 is 6 , the size of the manufacturer 2 is 2 .

As the Table2 shows, when the consumer environmental awareness is within a certain range $(\tau \in[0,4.69))$, the emission reduction rate is meaningful. In this range, retailer price per unit of manufacturer, emission reduction rate of product and the manufacturers' profit increase with $\tau$. 
Table 2. Impact of changes in consumer environmental awareness on manufacturers.

\begin{tabular}{ccccccc}
$\tau$ & $p_{1}$ & $p_{2}$ & $e_{1}$ & $e_{2}$ & $\pi_{1}$ & $\pi_{2}$ \\
\hline 0 & 6.667 & 6.667 & 0.600 & 0.200 & 302.444 & 126.444 \\
1 & 6.944 & 6.702 & 0.669 & 0.267 & 305.971 & 126.693 \\
2 & 7.273 & 6.781 & 0.746 & 0.336 & 309.838 & 127.064 \\
3 & 7.666 & 6.905 & 0.830 & 0.407 & 314.120 & 127.529 \\
4 & 8.137 & 7.075 & 0.926 & 0.483 & 318.908 & 128.046 \\
\hline
\end{tabular}

Therefore, manufacturers can improve the environmental awareness of consumers in this scope to make enterprises obtain higher profits and it is more conducive to reduce carbon emissions and protect the environment.

\section{Example 2}

In this subsection,we study the impact of the sensitivity of switchovers toward price on the emission reduction rates, retailer price per unit of manufacturer and profits of the two manufacturers. In table 3 , we assume that the consumer environmental awareness $\tau=2$, the size of the manufacturer 1 $M_{1}$ is 6 , the size of the manufacturer $2 M_{2}$ is 2 .

Table 3. Impact of changes in the sensitivity of switchovers toward price on manufacturers.

\begin{tabular}{lcccccc}
\hline$\theta$ & $p_{1}$ & $p_{2}$ & $e_{1}$ & $e_{2}$ & $\pi_{1}$ & $\pi_{2}$ \\
\hline 0 & 5.714 & 5.306 & 0.714 & 0.306 & 290.000 & 109.592 \\
0.2 & 6.241 & 5.795 & 0.724 & 0.316 & 296.173 & 114.911 \\
0.4 & 6.889 & 6.411 & 0.738 & 0.328 & 304.505 & 122.277 \\
0.5 & 7.273 & 6.781 & 0.746 & 0.336 & 309.838 & 127.064 \\
0.7 & 8.209 & 7.692 & 0.764 & 0.354 & 324.035 & 139.983 \\
0.9 & 9.466 & 8.928 & 0.789 & 0.379 & 345.817 & 160.114 \\
1 & 10.274 & 9.726 & 0.806 & 0.395 & 361.443 & 174.704 \\
\hline
\end{tabular}

As the Table3 shows, retail price,emission reduction rate of product and the expected manufacturer's profit increase with $\theta$. The profits of the two manufacturers increase much larger with the $\theta$.

In this case, manufacturers may increase their emission reduction rates to attract consumers as $\theta$ increases. The cost of reducing emissions increase with $e$, so the two manufacturers increase retail prices, then the profits of the two manufacturers increase with $\theta$.

\section{Example 3}

In this subsection,we study the impact of the size of the manufacturer on the emission reduction rates, retail price and profits of the two manufacturers. In table 4 , we assume that the $\tau=2, \theta=0.5$.

Table 4. Impact of changes in the size of the manufacturer on manufacturers.

\begin{tabular}{lccccccc}
\hline$M_{1}$ & $M_{2}$ & $p_{1}$ & $p_{2}$ & $e_{1}$ & $e_{2}$ & $\pi_{1}$ & $\pi_{2}$ \\
\hline 2 & 5 & 6.809 & 7.178 & 0.336 & 0.6436 & 141.719 & 298.703 \\
3 & 5 & 6.904 & 7.150 & 0.438 & 0.6430 & 171.213 & 262.600 \\
4 & 5 & 6.999 & 7.122 & 0.540 & 0.6424 & 193.789 & 234.435 \\
5 & 5 & 7.095 & 7.095 & 0.642 & 0.6420 & 211.827 & 211.827 \\
6 & 5 & 7.190 & 7.067 & 0.744 & 0.6413 & 226.842 & 193.261 \\
7 & 5 & 7.285 & 7.039 & 0.846 & 0.6408 & 239.845 & 177.726 \\
8 & 5 & 7.380 & 7.012 & 0.948 & 0.6402 & 251.534 & 164.524 \\
\hline
\end{tabular}

As the Table 4 shows,the two manufacturers' retailer price, emission reduction rates and the profits are equal when the two manufacturers are the same size. When the size of the manufacturer 1 increases gradually, but the size of the manufacturer 2 does not change, the retail price, the emission reduction rate and the profit of manufacturer 1 increase, and the manufacturer2's retail price, emission reduction rate and profit decrease accordingly.

We only study the size of the manufacturer 2 unchanged and the size of the manufacturer 1 change due to the symmetry of the two manufacturers. As the Table 4 shows, manufacturers who try to gain greater profits may expand their own business scale compared to the competitor. 


\section{Conclusions}

This study examines the influence of emission reduction rate, carbon quota, consumers' environmental awareness (CEA), manufacturer competition on retail prices, emission reduction rates and manufacturers' profits.

Our study has three main managerial implications.First,our results show that retail price, emission reduction rate of product and the manufacturers' profits increase with CEA. Manufacturers could improve CEA by advertising or other ways to obtain more profits. Second, retail prices, emission reduction rates and manufacturers' profits increase with price sensitivity. Manufacturers have to improve their competitiveness to the extent possible to achieve higher profits. Third, the two manufacturers' retail prices, emission reduction rates and profits are equal when the two manufacturers are the same size. Larger manufacturers will get higher profits. Therefore, manufacturers could try to expand their own business scale compared to their competitor.

\section{Acknowledgements}

This work was supported by the National Nature Science Foundation of China [grant numbers 71602103]; the Shandong Normal University [grant numbers 304132 and 15SQR017]. The usual disclaimer applies.

\section{References}

[1] IPCC, Climate Change Synthesis Report 2007: Comprehensive Report, IPCC, Geneva, Switzerland (2007)

[2] L.Zhang, J.Wang and J.You:Consumer environmental awareness and channel coordination with two substitutable products. J. European Journal of Operational Research. 241(1), 63--73(2015)

[3] R.Bemporad, Baranowski, M: Conscious Consumers Are Changing the Rules of Marketing. Are You Ready? Highlights from the BBMG Conscious Consumer Report, http://www.bbmg.com(2007)

[4] J.Li, W.Du, F.Yang,et al: The Carbon Subsidy Analysis in Remanufacturing Closed-Loop Supply Chain. J. Sustainability. 6(6),3861--3877(2014)

[5] D.Yang, T.Xiao:Pricing and green level decisions of a green supply chain with governmental interventions under fuzzy uncertainties.J.Journal of Cleaner Production. 149,1174--1187(2017)

[6] Y.Xu, H.Xie:Consumer Environmental Awareness and Coordination in Closed-Loop Supply Chain.J.Open Journal of Business \& Management.04(3),427--438(2016)

[7] L.Zhang, J.Zhang:Optimal Subsidy and Tax Policies for Green Product with Consumer Environmental Awareness.C. Hawaii International Conference on System Sciences. (2017)

[8] Z.Liu, Anderson, T.D, Cruz, J.M:Consumer environmental awareness and competition in two-stage supply chains.J.European Journal of Operational Research.218(3), 602--613. (2012)

[9] Q.Zhang, W.Tang and J.Zhang:Green supply chain performance with cost learning and operational inefficiency effects.J. Journal of Cleaner Production.112,3267--3284(2016)

[10] X.Chen, Z.Luo and X.Wang:Impact of efficiency, investment, and competition on low carbon manufacturing.J. Journal of Cleaner Production.143,388--400(2017)

[11] Zhu, W, \& He, Y: Green product design in supply chains under competition. European Journal of Operational Research, 258(1),165-180(2017) 


\section{Appendix}

\section{Proof of Theorem 1}

According to formula (3) and formula (4),

$$
\begin{aligned}
& \pi_{1}=p_{1}\left(a-p_{1}+\theta p_{2}+\tau e_{1}-\tau \theta e_{2}\right)-c e_{1}^{2}+\left(\frac{M_{1}}{M_{1}+M_{2}} s_{0}-M_{1}\left(1-e_{1}\right)\right) c_{0} . \\
& \pi_{2}=p_{2}\left(a-p_{2}+\theta p_{1}-\tau \theta e_{1}+\tau e_{2}\right)-c e_{2}^{2}+\left(\frac{M_{2}}{M_{1}+M_{2}} s_{0}-M_{2}\left(1-e_{2}\right)\right) c_{0} .
\end{aligned}
$$

we can conclude that:

$\frac{\partial^{2} \pi_{1}}{\partial e_{1}^{2}}=\frac{\partial^{2} \pi_{2}}{\partial e_{2}^{2}}=-2 c$

Because cost coefficient of emission reduction of the manufacturer satisfy $c \geq 0$, we have: $-2 c \leq 0$

We can draw a conclusion: two manufacturers' profit on the reduction rate is a concave function, there are optimal emission reduction rate $e_{1}^{*}$ and $e_{2}^{*}$ to make two manufacturers earns the largest income respectively.

Let $\frac{\partial \pi_{1}}{\partial e_{1}}=0, \frac{\partial \pi_{2}}{\partial e_{2}}=0$,thus we obtain Theorem 1 .

\section{Proof of Proposition 1.}

According to Formula $\quad e_{1}^{*}=\frac{p_{1} \tau+M_{1} c_{0}}{2 c} \quad e_{2}^{*}=\frac{p_{2} \tau+M_{2} c_{0}}{2 c}$

We have $\frac{\partial e_{1}^{*}}{\partial \tau}=\frac{p_{1}}{2 c}>0, \frac{\partial e_{2}^{*}}{\partial \tau}=\frac{p_{2}}{2 c}>0, \frac{\partial e_{1}^{*}}{\partial c_{0}}=\frac{M_{1}}{2 c}>0, \frac{\partial e_{2}^{*}}{\partial c_{0}}=\frac{M_{2}}{2 c}>0$,

thus we obtain Proposition 1.

\section{Proof of Proposition 2.}

According to Formula

$$
\begin{aligned}
& \pi_{1}^{*}=p_{1}\left(a-p_{1}+\theta p_{2}-\tau \theta \frac{p_{2} \tau+M_{2} c_{0}}{2 c}\right)+\frac{\left(p_{1} \tau+M_{1} c_{0}\right)^{2}}{4 c}+s_{0} c_{0} \frac{M_{1}}{M_{1}+M_{2}}-M_{1} c_{0} . \\
& \pi_{2}^{*}=p_{2}\left(a-p_{2}+\theta p_{1}-\tau \theta \frac{p_{1} \tau+M_{1} c_{0}}{2 c}\right)+\frac{\left(p_{2} \tau+M_{2} c_{0}\right)^{2}}{4 c}+s_{0} c_{0} \frac{M_{2}}{M_{1}+M_{2}}-M_{2} c_{0} .
\end{aligned}
$$


Let $\frac{\partial \pi_{i}^{*}}{\partial \tau}=0$

We have: $\tau=\frac{\left(M_{j} \theta-M_{i}\right) c_{0}}{p_{i}-2 p_{j} \theta}(i, j=1,2 ; i \neq j)$

When $\frac{\partial \pi_{i}^{*}}{\partial \tau} \leq 0$, is equivalent to $\tau \leq \frac{\left(M_{j} \theta-M_{i}\right) c_{0}}{p_{i}-2 p_{j} \theta}(i, j=1,2 ; i \neq j)$, The profit function of manufacturer $\mathrm{i}$ is the reduction function.

When $\frac{\partial \pi_{i}^{*}}{\partial \tau} \geq 0$, is equivalent to $\tau \geq \frac{\left(M_{j} \theta-M_{i}\right) c_{0}}{p_{i}-2 p_{j} \theta}(i, j=1,2 ; i \neq j)$, The profit function of manufacturer $i$ is the increment function.

The Proposition 2 can be obtained

\section{Proof of Proposition 3.}

According to Formula

$$
\begin{aligned}
& \pi_{1}^{*}=p_{1}\left(a-p_{1}+\theta p_{2}-\tau \theta \frac{p_{2} \tau+M_{2} c_{0}}{2 c}\right)+\frac{\left(p_{1} \tau+M_{1} c_{0}\right)^{2}}{4 c}+s_{0} c_{0} \frac{M_{1}}{M_{1}+M_{2}}-M_{1} c_{0} . \\
& \pi_{2}^{*}=p_{2}\left(a-p_{2}+\theta p_{1}-\tau \theta \frac{p_{1} \tau+M_{1} c_{0}}{2 c}\right)+\frac{\left(p_{2} \tau+M_{2} c_{0}\right)^{2}}{4 c}+s_{0} c_{0} \frac{M_{2}}{M_{1}+M_{2}}-M_{2} c_{0} .
\end{aligned}
$$

Thus:

$\frac{\partial \pi_{1}^{*}}{\partial \theta}=p_{1} p_{2}-p_{1} \tau \frac{p_{2} \tau+M_{2} c_{0}}{2 c} \quad \frac{\partial \pi_{2}^{*}}{\partial \theta}=p_{1} p_{2}-p_{2} \tau \frac{p_{1} \tau+M_{1} c_{0}}{2 c}$

Let $\frac{\partial \pi_{1}^{*}}{\partial \theta} \geq 0, \frac{\partial \pi_{2}^{*}}{\partial \theta} \geq 0$.The Proposition 3 can be obtained.

\section{Proof of Theorem 2}

According to Formula ( 6 ) and ( 7 ),

$$
\begin{aligned}
& \pi_{1}^{*}=p_{1}\left(a-p_{1}+\theta p_{2}-\tau \theta \frac{p_{2} \tau+M_{2} c_{0}}{2 c}\right)+\frac{\left(p_{1} \tau+M_{1} c_{0}\right)^{2}}{4 c}+s_{0} c_{0} \frac{M_{1}}{M_{1}+M_{2}}-M_{1} c_{0} . \\
& \pi_{2}^{*}=p_{2}\left(a-p_{2}+\theta p_{1}-\tau \theta \frac{p_{1} \tau+M_{1} c_{0}}{2 c}\right)+\frac{\left(p_{2} \tau+M_{2} c_{0}\right)^{2}}{4 c}+s_{0} c_{0} \frac{M_{2}}{M_{1}+M_{2}}-M_{2} c_{0} .
\end{aligned}
$$


Let $\frac{\partial \pi_{1}^{*}}{\partial p_{1}}=0, \frac{\partial \pi_{2}^{*}}{\partial p_{2}}=0$, The theorem can be obtained. 